\title{
RESISTANCE OF KLEBSIELLA PNEUMONIAE CLINICAL ISOLATES: LINKAGE OF OUTER MEMBRANE PROTEINS (OMPS) WITH PRODUCTION ESBLS
}

\author{
Lívia Érika Carlos Marques, Danielle Ferreira de Oliveira, Márcia Maria Mendes Marques, Ana Raquel Araújo da Silva, \\ Carlucio Roberto Alves, Maria Izabel Florindo Guedes*
}

Laboratório de Bioquímica Humana, Departamento de Nutrição, Universidade Estadual do Ceará, Fortaleza, CE, Brasil.

Submitted: April 24, 2010; Returned to authors for corrections: August 30, 2010; Approved: January 13, 2011.

\begin{abstract}
Three isolates of Klebsiella pneumoniae, collected from the University Hospital in Fortaleza, Brazil, were analyzed to determine their resistance to multiple antibiotics. The results of this study showed that the resistance of the clinically isolated bacteria is associated with the production of extended-spectrum betalactamases (ESLBs) and loss of outer membrane proteins.
\end{abstract}

Key words: Klebsiella pneumoniae, $\beta$-lactamase and porin.

Klebsiella pneumoniae (Enterobacteriaceae) is an opportunist human pathogen that accounts for a significant proportion of hospital-acquired urinary tract infections, pneumonia, septicaemia, and soft tissue infections. Due to its ability to spread rapidly in a hospital environment and resistance to multiple drugs, this strain is for nosocomial outbreaks world-wide, generally associated with high morbidity and mortality $(5,6)$.

One of the most prevalent mechanisms of bacterial resistance among Gram-negative bacteria is the production of $\beta$-lactamases. Extended-spectrum $\beta$-lactamases (ESBLs) are a group of clinically very important $\beta$-lactamases because they are able to hydrolyze the extended-spectrum cephalosporins (ceftazidime, cefotaxime, ceftriaxone) and monobactans (aztreonam) (2). Frequently, bacterial species will not only possess a $\beta$-lactamase, but also exhibit porin deletion and thus resistance derives from a synergy between reduced permeability and $\beta$-lactamase activity (8). The outer-membrane proteins (OMPs) of Enterobacteriaceae have medical importance because its constituents play major roles in the permeability of antimicrobial agents and substrates, and in interactions with the host defense mechanisms (3).

The aim of the present study was to evaluate the relationship between the resistances of ESBL-expressing $K$. pneumoniae clinical isolates and lost outer membrane proteins.

The $K$. pneumoniae isolates were collected from hospitalized patients in the Walter Cantídio University Hospital, Fortaleza/Brazil, in June of 2005. The strains were identified by standard biochemical methods and $K$. pneumoniae ATCC 4352 and E.coli ATCC 25922 was used as the reference strain. The strains were maintained as frozen stocks at $-70^{\circ} \mathrm{C}$ in the presence of $15 \%$ of glycerol and cultured in brain heart infusion (BHI) agar (Difco) for 24 hours at $37^{\circ} \mathrm{C}$ for later analysis. Antimicrobial susceptibility to cephalotin, cefoxitin, 
ceftriaxone, cefotaxime, amoxillin/clavulanate cefepime and aztreonam was performed by disk diffusion method according to Clinical and Laboraty Standards Institute (CLSI) recommendations (7). All tests were carried out three times. The isolates were further examined by the double-disk synergy test for ESBLs detection as previously described by Vercauteren et al. (9), based on the inhibition toward the disk containing the $\beta$-lactamase inhibitor clavulanate.

Electrophoretic analysis of $K$. pneumoniae OMPs was carried out using the buffer system of Laemmli (4). Bacterial cultures were grown at $37^{\circ} \mathrm{C}$ overnight in $\mathrm{BHI}$ agar medium. The cells were collected by centrifugation $(13000 \mathrm{~g}$ for $3 \mathrm{~min}$.) of $1 \mathrm{~mL}$ of culture and suspended in $100 \mu 1$ of sample buffer. The cells suspensions were boiled at $100^{\circ} \mathrm{C}$ for 10 minutes. In addition, 20 $\mu l$ of the protein were used for SDS-PAGE on a vertical slab gel, containing $4 \%$ stacking gel and a $17 \%$ separating gel. Protein bands were visualized by Coomassie brilliant blue staining. Molecular weights were estimated by comparison with the protein molecular weight markers MW-SDS-70L (Sigma-Aldrich Co., USA), ranging in size from $14,4-94,0 \mathrm{kDa}$. The protein concentration of bacterial suspension was determined by the Bradford essay (1).

Table 1 displays the results of antimicrobial susceptibility of K. pneumoniae isolates. Kp 3, Kp 6 and $\mathrm{Kp} 8$ were resistant to third and fourth generation cephalosporins and monobactans. Application of the double-disk synergy test procedure also showed that these isolate ESBL production. This data confirmed the observation of Lopes et al., (5) that K. pneumoniae is resistant to a number of antibiotics, mainly extended-spectrum cephalosporins and penicillins, due to acquisition of plasmids that encode for the production of extended-spectrum $\beta$-lactamases.

Table 1. Antimicrobial susceptibility of $K$. pneumoniae clinical isolates and reference strain

\begin{tabular}{ll}
\hline Strains & Resistance phenotypes \\
\hline E. coli $($ ATCC 25922) & \\
Kp (ATCC 4352) & - \\
Kp 8 & KF, CRO, ATM, AMC/CAC, CTX, \\
Kp 6 & KF, CRO, FOX, ATM, FEP \\
Kp 3 & KF, CRO, FOX, ATM, FEP, AMC/CAC, CTX, \\
\hline - not resistance; KF-cephalotin, FOX-cefoxitin, CRO-ceftriaxone, CTX-cefotaxime, FEP-cefepime, ATM-aztreonam, AMC/CAC-amoxicilin/clavulanate.
\end{tabular}

SDS-PAGE analysis (Fig. 1) of the $K$. pneumoniae isolates revealed several proteins bands in the 30 to $66 \mathrm{kDa}$ range. The results of this study indicate that Kp3, Kp 6 and Kp 8 strains, resistant to aztreonam and all cephalosporins (Table 1) to loss one porin. However, we can say that this porin is $30-43 \mathrm{kDa}$ range, suggesting that it may be OmpK35 or OmpK36. It has been clearly demonstrated that porin deficiency contributes to increasing the level of resistance to ESBLs-producing strains (3).

In conclusion, this data suggests that resistance to $K$. pneumoniae clinical isolates is due to the association between extended-spectrum beta-lactamases (ESLBs)-producing with lost outer membrane proteins. Further studies, using techniques of molecular biology will be developed with the intention of confirming production ESBLs.

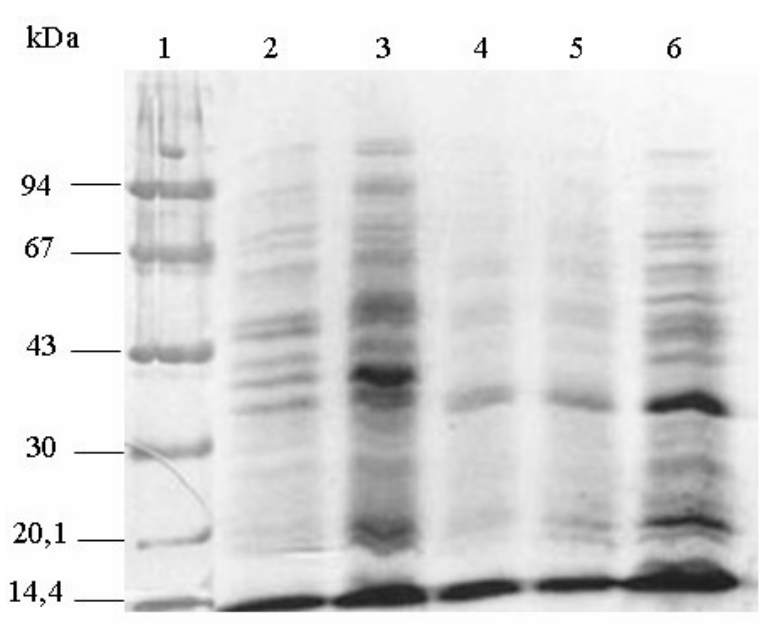

Figure 1. SDS-PAGE analysis of Klebsiella pneumoniae clinical isolates. Molecular sizes (lane 1), E. coli ATCC 25922 (lane 2), Kp ATCC 4352 (lane 3), Kp 08 (lane 4), Kp 06 (lane 5), Kp 03 (lane 6) 


\section{ACKNOWLEDGEMENTS}

The authors would like to thank the following Brazilian government agencies for their financial support: Fundação Coordenação de Aperfeiçoamento de Pessoal de Nível Superior (CAPES) and Fundação de Amparo a Pesquisa do Estado do Ceará (FUNCAP).

\section{REFERENCES}

1. Bradford, M. M. (1976). A rapid and sensitive method for the quantitation of microgram quantities of protein utilizing the principle of protein-dye binding. Anal. Biochem. 72, 248-254.

2. Freitas, A.L.P.; Machado, D.P.; Soares, F.S.C. e Barth, A.L. (2003) Extended-Spectrum $\beta$-Lactamases in Klebsiella spp and Escherichia coli obtained in a brazilian teaching hospital: detection, prevalence and molecular typing. Brazilian J Microbiol. 34, 344-348.

3. Hernández-Allés, S.; Albertí, S.; Alvarez, D.; Dosmenech-Sanchez, A.; Martinez-Martinez, L.; Gil, J.; Tomas, J. M.; Benedí, V. J. (1999). Porin expression in clinical isolates of Klebsiella pneumoniae. Microbiol. 145, 673-679.
4. Laemmli, U.K. (1970). Cleavage of structural proteins during the assembly of the head of bacteriophage T4. Nature. 227, 680-685.

5. Lopes, A.C.S.; Rodrigues, J.F.; Junior, M.A.M. (2005). Molecular Typing of Klebsiella pneumoniae isolates from public hospitals in Recife, Brazil. Microbiol. Res. 160, 37-45.

6. Martinez-Martinez, L.; Hernandez-Alles, S.; Albertí, S.; Tomás, J.M.; Benedi, V.J.; Jacoby, G.A. (1996). In Vivo Selection of Porin-Deficient Mutants of Klebsiella pneumoniae with Increased Resistance to Cefoxitin and Expanded-Spectrum Cephalosporins. Antimicrob. Agents Chemother. 40, 342-348.

7. National Committee for Clinical Laboratory Standards. (2000). Performance standards for antimicrobial disk susceptibility testing. In: Approved Standards M2-A7, seventh ed. National Committee for Clinical Laboratory Standards, Wayne, PA.

8. Turner, P.J. (2005). Extended-Spectrum $\beta$-Lactamases. Clin. Infectiosas Dis. 41, 273-275.

9. Vescauteren, E.; Descheemaeker, P.; Ieven, M.; Sanders, C. C.; Goossens, H. (1997). Comparison of screening methods for detection of extended spectrum $\beta$-lactamases and their prevalence among blood isolates of Escherichia coli and Klebsiella spp. in a Belgian teaching hospital. J Clin Microbiol 35, 2191-2197. 